\title{
BMJ Open Expectation-induced enhancement of pain, itch and quality of life in psoriasis patients: study protocol of a randomised controlled trial
}

\author{
Stefanie Hölsken, ${ }^{1}$ Frederik Krefting, ${ }^{2}$ Manfred Schedlowski, ${ }^{1,3}$ \\ Wiebke Sondermann (iD ${ }^{2}$
}

To cite: Hölsken S,

Krefting F, Schedlowski M, et al. Expectation-induced enhancement of pain, itch and quality of life in psoriasis patients: study protocol of a randomised controlled trial. BMJ Open 2021;11:e047099. doi:10.1136/ bmjopen-2020-047099

- Prepublication history and additional supplemental material for this paper are available online. To view these files, please visit the journal online (http://dx.doi.org/10.1136/ bmjopen-2020-047099).

Received 19 November 2020 Accepted 16 August 2021

Check for updates

(C) Author(s) (or their employer(s)) 2021. Re-use permitted under CC BY-NC. No commercial re-use. See rights and permissions. Published by BMJ.

${ }^{1}$ Institute of Medical Psychology and Behavioral Immunobiology, University Hospital Essen, University Duisburg-Essen, Essen, Germany

${ }^{2}$ Department of Dermatology, Venereology and Allergology, University Hospital Essen, University Duisburg-Essen, Essen, Germany

${ }^{3}$ Department of Clinical Neuroscience, Osher Center for Integrative Medicine, Karolinska Institute, Stockholm, Sweden

Correspondence to Dr. Wiebke Sondermann; wiebke.sondermann@uk-essen. de

\section{ABSTRACT}

Introduction Experimental and clinical data demonstrate that skin diseases like psoriasis are affected by psychological factors and can be modulated by interventions other than conventional drug therapy. The expectation of patients towards the benefit of a forthcoming treatment as well as treatment preexperiences have been demonstrated as crucial factors mediating placebo responses in inflammatory skin diseases. However, it is unknown whether and to what extent treatment outcomes of psoriasis patients under therapy with monoclonal antibodies like secukinumab can be experimentally modulated at subjective and physiological levels by modifying the expectation of patients via verbal instruction or prior experience. Methods and analysis Treatment expectations will be modulated in patients with moderate-to-severe psoriasis undergoing treatment with the anti-interleukin17A monoclonal antibody secukinumab. Patients with a Psoriasis Area and Severity Index (PASI) $>12$ will be randomly allocated to one of three groups ( $\mathrm{N}=40$ each). As a standard schedule, patients in the pharmacological control group (group 1) will be treated weekly with $300 \mathrm{mg}$ secukinumab, while patients in groups 2 and 3 will receive only $75 \mathrm{mg}$ secukinumab ( $75 \%$ dose reduction) during all treatment weeks. In addition to the injections, patients in group 3 will ingest a novel tasting drink, with a cover story explaining that previous studies showed additional beneficial effects of this combination (drug and drink). Patients will be assessed and treated at nine visits over a 16-week period, during which the severity of pain and itch symptoms, skin lesions and quality of life will be analysed with standardised questionnaires and the PASI.

Ethics and dissemination This study was approved by the Ethics committee of the Medical Faculty of the University Duisburg-Essen. Study outcomes will be published in peer-reviewed scientific journals.

\section{INTRODUCTION}

Drugs do not act in a vacuum. In any medical treatment, the drug's biological effect depends on an interaction of the drug's pharmacological performance and, among other things, the individual's psychological predisposition. This psychological predisposition is

\section{Strengths and limitations of this study}

- This study will provide first insights in how far modulation of patients' expectation will affect efficacy of pharmacological treatment in patients with inflammatory skin diseases like psoriasis.

- The results of the study will help to gain knowledge of how and via which mechanisms expectations of psoriasis patients can be framed and modulated to increase the therapeutic outcome for the patients' benefit. In addition, these data might provide a basis for extrapolating these approaches to treatment strategies in patients with other chronic inflammatory diseases.

- The selection of comprehensive outcome measures, including subjective and objective clinical as well as neuroendocrine markers, assures precise analyses of study endpoints.

- The primary limitation of this study is a potentially increased drop-out rate in the dose-reduction control group due to suboptimal therapy response.

influenced by numerous factors and in particular, by the patient's expectation of a medication's beneficial effects, which is mainly steering the placebo effect. ${ }^{1-3}$ Our knowledge about the neurobiological and neuropsychological mechanisms mediating placebo responses has advanced substantially during the last two decades and current experimental and clinical data indicate that the systematic manipulation of patients' expectations can affect the outcome of medical interventions in general and pharmacological treatment in particular. ${ }^{4-6}$ However, whether and to what extent this holds true for chronic inflammatory skin diseases and the treatment with systemic therapies, such as monoclonal antibodies, remains unclear. Thus, the presented clinical proof-of-concept trial will systematically modulate treatment expectation in psoriasis patients undergoing monoclonal antibody treatment and investigate the effects 
of expectation on treatment outcomes at the subjective and physiological level.

Psoriasis is a chronic inflammatory skin disorder affecting around $2 \%$ of the Western populations. ${ }^{78}$ Patients suffer from severe aching skin discomfort and pruritus. Owing to the visibility of the typical erythematous, scaly patches and plaques, psoriasis is highly stigmatising and can lead to embarrassment, shame, lowered self-esteem, social anxiety and retreat from social contacts. ${ }^{9}$ Additionally, psoriasis patients carry an increased risk of developing cardiovascular and metabolic diseases, as well as psychiatric disorders. ${ }^{10-12}$ As a result of these highly burdening symptoms, patients with psoriasis have a reduction in their quality of life (QoL) similar to or worse than patients with other chronic diseases, such as ischaemic heart disease and diabetes. ${ }^{13} 14$ Clinical observations and experimental evidence suggest that inflammatory skin diseases are affected by psychological factors, such as stress and anxiety ${ }^{1516}$ and that patients' expectations of treatment outcome are a crucial factor mediating placebo responses (for reviews see, refs. 217 18). This patient group, therefore, provides an ideal clinical model to investigate the effect of treatment expectation in the context of a somatic disease at the subjective (eg, pain and itching) and objective level (eg, skin lesions).

Within the last years, research on the pathophysiology of psoriasis has seen substantial advances. ${ }^{19}{ }^{20}$ Recent data revealed a central pathogenetic role for Th17 cells in psoriatic skin inflammation. ${ }^{21}$ Today, the so-called interleukin (IL)-23/Th17 immune axis is regarded as the major immune pathway in the pathophysiology of psoriasis. ${ }^{192}$ The first IL-17A inhibitor for the treatment of moderate-to-severe plaque psoriasis, secukinumab, a human monoclonal antibody, was approved in 2015. ${ }^{22}$ It has been shown to effectively suppress the inflammatory response and to reduce disease burden, symptoms such as pain and itch and to increase QoL in psoriasis. ${ }^{23-25}$ However, biologics such as secukinumab may provoke side effects, such as infections, and are associated with high treatment costs. ${ }^{2627}$

In the presented trial, the effects of verbally induced expectations on the severity of pain and itch symptoms, QoL as well as skin lesions in patients suffering from moderate-to-severe psoriasis will be investigated during treatment with secukinumab. The induction of expectations will be combined with a reduction in medication dose. In the context of associative learning, placeboinduced dose reduction has already been shown to be an effective method to maintain drug efficacy while reducing the amount of medication required. ${ }^{28} 29$

With regard to the outcome measures, pain is the best-studied one regarding contributing factors and underlying mechanisms, as it has been a central topic in placebo research from its beginnings. ${ }^{30}$ Placebo analgesia was shown to be effective in healthy volunteers as well as patient groups (Forsberg $\left.e t a l^{11}, 2017\right)$ and to be related to an activation of the body's own opioid system and other non-opioid systems. ${ }^{32}$ Itch on the other hand has received less attention in research. It was found to be susceptible to verbal suggestion in healthy volunteers ${ }^{33} 34$ and observational learning in patients ("contagious itch ${ }^{, 35}$ ). Data from pharmacological trials including placebo arms suggest that chronic itch is susceptible to placebo interventions as well. ${ }^{18} \mathrm{QoL}$, as an important outcome measure for any medical treatment, has already been shown to be influenced by patients' expectations in various conditions. ${ }^{36-38}$ Next to these subjective measures, a central aspect to psoriasis is the objective extent of skin lesions. A recent meta-analysis of trials addressing the effectiveness of systemic treatments found that improvements in Psoriasis Area and Severity Index (PASI) scores were relatively small in the placebo arms. ${ }^{39}$ The effects of patients' expectations on treatment outcome have not been assessed experimentally in psoriasis patients for any of these measures so far.

\section{METHODS AND ANALYSIS \\ Design}

This proof-of-concept study will employ a randomised factorial design. ${ }^{40}$ Objective and subjective signs and symptoms of psoriasis will be analysed in patient groups receiving either standard pharmacological treatment (=treatment as usual (TAU), $100 \%$ drug dose) or treatment with a drug dose reduced by $75 \%$. Patients in the dose reduction group will be further divided into two groups, differing in the degree of treatment expectation. Allocation to one of the three groups will be randomised $(\mathrm{N}=40$ each). The study flow is depicted in figure 1 . As a standard schedule, patients in the pharmacological control group (group 1; TAU) will be treated weekly with $300 \mathrm{mg}$ secukinumab (as in clinical routine consisting of two subcutaneous injections with $150 \mathrm{mg}$ ) for 5 weeks (weeks 0-4), followed by two additional injections four (week 8) and eight (week 12) weeks later. Group 2 (Expectation-LOW) will serve as a dose reduction control group receiving one injection with $75 \mathrm{mg}$ secukinumab and one injection with $\mathrm{NaCl}$ (sodium chloride) during all treatment weeks. The reduced dosage of $75 \mathrm{mg}$ in groups 2 and 3 was chosen since the resulting difference between the groups is expected to be pronounced enough to detect significant differences (approximately 20\% points with regard to PASI75 response in week 12) but the expected response is still sufficient to avoid high drop-out rates due to a premature termination by the participants. ${ }^{23}$ Gr Group 3 (Expectation-HIGH) will be treated with the dose reduction regimen as group 2. However, patients in this group will receive an additional positive verbal instruction presented in a standardised manner by the main study physician to strengthen their expectation towards the benefits of the treatment. The salience of this verbal instruction will be reinforced by combining each injection with the ingestion of a newly tasting beverage together with detailed information about the potential beneficial effects of this combination (drug and drink). The gustatory stimulus was added since recent studies 


\begin{tabular}{|c|c|c|c|c|c|c|c|c|c|}
\hline \multirow[b]{3}{*}{ TIMEPOINT } & \multicolumn{9}{|c|}{ Study-Period } \\
\hline & \multirow{2}{*}{$\begin{array}{c}\begin{array}{c}\text { Pre- } \\
\text { Check }\end{array} \\
-t_{1}\end{array}$} & \multicolumn{7}{|c|}{ Treatment } & \multirow{2}{*}{$\begin{array}{c}\text { Follow- } \\
\text { up } \\
t_{4}\end{array}$} \\
\hline & & $t_{1}$ & $t_{2}$ & $t_{3}$ & 4 & $t_{5}$ & $t_{6}$ & t, & \\
\hline & & $\begin{array}{c}\text { Week } \\
0\end{array}$ & $\begin{array}{c}\text { Week } \\
1\end{array}$ & $\begin{array}{c}\text { Week } \\
2\end{array}$ & $\begin{array}{c}\text { Week } \\
3\end{array}$ & $\begin{array}{c}\text { Week } \\
4\end{array}$ & $\begin{array}{c}\text { Week } \\
8\end{array}$ & $\begin{array}{c}\text { Week } \\
12\end{array}$ & $\begin{array}{c}\text { Week } \\
16\end{array}$ \\
\hline \multicolumn{10}{|l|}{ ENROLMENT: } \\
\hline Eligibility screen & $x$ & & & & & & & & \\
\hline Informed consent & $x$ & & & & & & & & \\
\hline Randomisation & & $x$ & & & & & & & \\
\hline \multicolumn{10}{|l|}{ INTERVENTIONS: } \\
\hline \multicolumn{10}{|l|}{$\begin{array}{l}\text { Group 1 } \\
\text { (Pharmacological } \\
\text { Control) }\end{array}$} \\
\hline \multicolumn{10}{|l|}{$\begin{array}{l}\text { Group } 2 \\
\text { (Dose-Reduction } \\
\text { "Expectation-Low") }\end{array}$} \\
\hline \multicolumn{10}{|l|}{$\begin{array}{l}\text { Group } 3 \\
\text { (Dose-Reduction } \\
\text { "Expectation-High") }\end{array}$} \\
\hline \multicolumn{10}{|l|}{ ASSESSMENTS: } \\
\hline $\begin{array}{l}\text { Baseline } \\
\text { Demographics }\end{array}$ & $x$ & & & & & & & & \\
\hline $\begin{array}{l}\text { Cortisol-Awakening } \\
\text { Response }\end{array}$ & $x$ & & & & & & & & \\
\hline Alpha-Amylase & $x$ & & & & & & & & \\
\hline cytokines & $x$ & & & & $x$ & $\mathrm{x}$ & $x$ & $x$ & $x$ \\
\hline Psoriasis Severity & $x$ & $\leftarrow$ & & & & & & $\longrightarrow$ & $x$ \\
\hline Pain & $x$ & $\curvearrowleft$ & & & & & & $\longrightarrow$ & $x$ \\
\hline Itch & $x$ & $\leftarrow$ & & & & & & $\longrightarrow$ & $x$ \\
\hline Quality of uife & $x$ & $\leftarrow$ & & & & & & $\longrightarrow$ & $x$ \\
\hline Side effects & $x$ & $\leftarrow$ & & & & & & $\longrightarrow$ & $x$ \\
\hline $\begin{array}{l}\text { Depression and } \\
\text { Anxiety }\end{array}$ & $x$ & $\leftarrow$ & & & & & & $\rightarrow$ & $x$ \\
\hline Perceived Stress & $x$ & & & & $x$ & $x$ & $x$ & $x$ & $x$ \\
\hline Personality Factors & $x$ & & & & & & & & \\
\hline
\end{tabular}

Figure 1 Schedule of enrolment, interventions and assessments. Adapted from the SPIRIT (Standard Protocol Items: Recommendations for Interventional Trials) 2013 template. ${ }^{59}$

demonstrated that the combination of drug and drink is able to evoke associative learning processes, and can be employed as a placebo-induced dose reduction strategy in immunopharmacological regimens. ${ }^{42} 43$ The verbal information regarding the brain-immune system communication will be visually underlined by a printed illustration. The English translation of the standardised verbal instruction can be found as an online supplemental material. Since we hypothesise that state and trait variables can modulate expectation per se and its effect on health outcome, we will identify neuroendocrine and psychological parameters that modulate an individual's effect of expectation on treatment outcome. All patients and the study physicians rating the skin symptoms will be blinded towards the drug and $\mathrm{NaCl}$ injections, as well as the study hypotheses. An unblinded team consisting of 
study physicians and coordinators will be responsible for the dispense and application of secukinumab/placebo.

\section{Patients}

Patients will be recruited via the outpatient department of the Department of Dermatology, Venereology and Allergology at the University Hospital Essen. Furthermore, the study will be advertised via (social) media and among dermatological practices. Patients' diagnosis of psoriasis will be confirmed by a board certified dermatologist. The main inclusion criteria regarding the severity of psoriasis are a PASI of at least 12 and a body surface area (BSA) affected by plaque psoriasis of more than $10 \%$. The decision for these thresholds was made based on the main inclusion criteria of most of the pivotal studies of secukinumab and other biologics. ${ }^{2344}$ Further, according to the German S3 guideline on psoriasis, systemic treatment is recommended in clinical routine when a patient reaches a BSA $>10$ and/or a PASI $>10$ and a Dermatology Life Quality Index (DLQI) $>10 .{ }^{45}$ Lastly, these thresholds allow for adequate objective comparisons between the status at baseline and under therapy. All inclusion and exclusion criteria are summarised in box 1 .

\section{Procedure and measures}

Study-specific measures will be performed at the Department of Dermatology, Venereology and Allergology at the University Hospital Essen. As in routine, blood samples (including differential blood count and clinical chemistry) will be taken before entering the study and every 4 weeks. The severity of pain and itch symptoms, skin lesions and QoL in patients will be assessed nine times from precheck to follow-up (see figure 1). During each visit to the study centre, patients will undergo a routine medical check and the pharmacological treatment will be monitored with regard to potential side effects. To this end, the Generic Assessment of Side Effects ${ }^{46}$ will be employed. Pain and itch symptoms will be assessed with visual rating scales, skin lesions and disease activity will be objectively quantified by the study physician with the PASI, and adverse effects on QoL due to the disease and the treatment will be analysed with the DLQI. ${ }^{47}$ Blood and saliva samples will be taken at five visits with a 4-week interval, to investigate relevant cytokines and cortisol levels. At the same instants, psychophysiological stress will be assessed with the Perceived Stress Scale- $10^{48}$, to be able to examine a relationship between these measures.

In addition, we will identify neuroendocrine and psychological parameters that modulate an individual's effect of expectation on treatment outcome. Salivary cortisol awakening response and salivary alpha-amylase activity will be assessed as markers of HPA (hypothalamuspituitary-adrenal) axis and sympathetic activity, respectively. ${ }^{4-51}$ Saliva samples will be obtained between study visits 1 and 2 on two consecutive days immediately after awakening as well 30 and 45 min after awakening. ${ }^{51}$ As an indication for the patients' mental state, anxious and depressive symptoms will be assessed using

\section{Box 1 Inclusion and exclusion criteria}

\section{Inclusion criteria}

1. Diagnosis of moderate-to-severe chronic plaque psoriasis for at least 6 months.

2. Psoriasis Area and Severity Index $>12$ and body surface area affected by plaque psoriasis $\geq 10 \%$.

3. Patient is a candidate for systemic therapy (this is defined as a patient having moderate-to-severe chronic plaque psoriasis that is inadequately controlled by topical treatment and/or phototherapy and/ or previous systemic therapy).

4. Women must not be pregnant and if of childbearing potential, must have a negative serum pregnancy test before entering the study.

5. Women of childbearing potential must agree to use a highly effective method of contraception throughout the therapy.

\section{Exclusion criteria}

1. Age $<18$ years.

2. Forms of psoriasis other than chronic plaque (eg, pustular, erythrodermic, guttate or drug-induced) psoriasis.

3. Existing contraindication to therapy with secukinumab according to the summary of product characteristics like history of hypersensitivity to secukinumab or its excipients or to drugs of similar chemical classes or clinically relevant (chronic or acute) infections, for example, untreated (latent) tuberculosis or HIV infection.

4. Significant medical problems, which in the opinion of the investigator significantly immunocompromise the subject and/or place the subject at unacceptable risk for receiving therapy with secukinumab.

5. History of, respectively active, inflammatory bowel disease.

6. Administration of live vaccines $<6$ weeks before first injection of secukinumab.

7. Any medical or psychiatric condition which, in the investigator's opinion, would preclude the participant from adhering to the protocol or completing the study per protocol.

8. Previous exposure to secukinumab.

9. Ongoing use of systemic antipsoriatic treatments (concomitant topical treatment is allowed).

10. History or evidence of ongoing alcohol or drug abuse, within the last 6 months before start of the study.

11. Subject is lacking capacity to consent.

the State-Trait-Anxiety-Depression-Inventory. ${ }^{52}$ Various personality traits will be assessed with the Behavioural Inhibition/Approach System Sensitivity scale, ${ }^{53}$ Somatosensory Amplification Scale, ${ }^{54}$ and Big Five Short Screener-10. ${ }^{55}$ Furthermore, previous treatment experience will be inquired using a numerical rating scale. To validate the experimental manipulation, the Treatment Expectation Questionnaire ${ }^{56}$ will be filled out both at the precheck and after 1 week of treatment.

The first patient was included into the study in November 2020. Data collection is planned to be terminated at the beginning of 2023.

\section{Randomisation}

Patients will be randomly allocated to one of the three groups by one of the study coordinators. Randomisation will be performed using computer-generated random numbers, with treatment groups being assigned to sequential participant numbers and blackened out until 
patient inclusion. To account for the possibility of dropouts being higher in group 2 than in the other two groups, data will be reviewed with regard to number of patients per treatment group after inclusion of 60 patients. If necessary, group allocation criteria will be adjusted to ensure equal group sizes.

\section{Sample size}

The sample size is based on an a priori power analysis with $G^{*}$ Power $\left(V \cdot 3.1 .9 .2^{57}\right)$ for main and interaction effects in a two-factorial analysis of variance (ANOVA) with the factors group and time. $\mathrm{G}^{*}$ Power is a versatile tool performing both post hoc power analyses as well as a priori determinations of required sample sizes. It allows the user to choose between the common statistical tests, including repeated measures designs. Assuming a mean effect size of $\mathrm{f}^{2}=0.3$ with regard to the main outcome parameters skin-related QoL, itch and skin pain (based on previous studies with a similar experimental design), significance level of $\alpha=0.05$ and power $(1-\beta)$ of 0.90 , the number of patients is $\mathrm{N}=84$. To account for possible $20 \%$ exclusions and drop-outs during the study and to allow for stratified recruitment of male and female patients, a group size of $\mathrm{N}=40$ patients (total $\mathrm{N}=120$ ) is planned.

\section{Hypotheses}

We hypothesise to detect differences among groups in our primary outcomes skin pain, itch and skin-related QoL. The subjective measures lesion severity as well as the immunological markers will be explored as secondary outcomes. We expect to observe clinical improvements in all three groups. However, we hypothesise that improvements will be greater in group 3 (Expectation-HIGH) compared with group 2 (Expectation-LOW). In addition, based on the proven effectiveness of the medication, we expect group 1 (TAU) to show more pronounced improvements compared with groups 2 and 3 .

\section{Data analysis}

Data will be analysed employing a linear mixed model/ repeated-measures ANOVA for each of the primary and secondary outcomes listed above. False discovery rate correction will be employed to adjust for multiple comparisons. The primary independent variables will be the treatment condition (between subjects; with the levels being pharmacological control, dose-reduction expectation low and dose-reduction expectation high) and time point of assessment (within subjects; with each of the nine visits as one factor level). Data on previous treatment experience and depressive symptoms as potential confounds will be collected to confirm equal dissemination among groups. In case groups are not balanced, these measures will be included as covariates in the statistical models. To validate the experimental manipulation, treatment expectation will be compared between treatment groups using a one-way repeated measures ANOVA. Interim analyses may be performed within the scope of doctoral theses, but will not have any influence on trial termination. Missing data will be handled employing regression imputation, if at least $80 \%$ of the data for a specific patient are present. Patients with more missing data on a certain outcome will be handled as drop-out for that outcome's analysis. ${ }^{58}$

\section{Patients and public involvement statement}

Patients and the public were not involved in the planning of this study. They will not be involved in the reporting or dissemination of results, but their feedback will be taken into account in the planning of potential follow-up studies.

\section{ETHICS AND DISSEMINATION}

The study was approved by the Ethics committee of the Medical Faculty of the University Duisburg-Essen (IRB protocol number 19-8636-BO). Study outcomes will be published in peer-reviewed scientific journals. All patients will be required to give their written informed consent for participation before the performance of any study specific measures. Consent will be obtained by the study physicians after informing patients about all study procedures and answering potential questions. After trial completion, all patients will get the chance to receive TAU with secukinumab, independently of prior group allocation. All data will be pseudonymised with a dedicated software. Decoding will only be possible for the associated study personnel. Identifiable data will be stored securely within the hospital in accordance with the European Union General Data Protection Regulation. The investigators plan to publish the study results in peer-reviewed scientific journals, with no restrictions to publication.

Contributors SH: precise planning of the study, drafting the manuscript, has given final approval of the version to be published. FK: precise planning of the study, revising the manuscript critically for important intellectual content, has given final approval of the version to be published. MS: initial idea, conception and design of the study, drafting the manuscript, revising the manuscript critically for important intellectual content, has given final approval of the version to be published. WS: initial idea, conception and design of the study, drafting the manuscript, revising the manuscript critically for important intellectual content, has given final approval of the version to be published.

Funding Gefördert durch die Deutsche Forschungsgemeinschaft (DFG) Projektnummer 422744262-TRR 289-Funded by the Deutsche Forschungsgemeinschaft (DFG, German Research Foundation)—Project-ID 422744262-TRR 289).

Competing interests WS reports grants from medi Bayreuth, personal fees from Janssen, grants and personal fees from Novartis, personal fees from Lilly UCB, Almirall, LEO Pharma and Sanofi Genzyme, outside the submitted work. The other authors declare that they have no competing interests.

Patient consent for publication Not required.

Provenance and peer review Not commissioned; externally peer reviewed.

Supplemental material This content has been supplied by the author(s). It has not been vetted by BMJ Publishing Group Limited (BMJ) and may not have been peer-reviewed. Any opinions or recommendations discussed are solely those of the author(s) and are not endorsed by BMJ. BMJ disclaims all liability and responsibility arising from any reliance placed on the content. Where the content includes any translated material, BMJ does not warrant the accuracy and reliability of the translations (including but not limited to local regulations, clinical guidelines, terminology, drug names and drug dosages), and is not responsible for any error and/or omissions arising from translation and adaptation or otherwise. 
Open access This is an open access article distributed in accordance with the Creative Commons Attribution Non Commercial (CC BY-NC 4.0) license, which permits others to distribute, remix, adapt, build upon this work non-commercially, and license their derivative works on different terms, provided the original work is properly cited, appropriate credit is given, any changes made indicated, and the use is non-commercial. See: http://creativecommons.org/licenses/by-nc/4.0/.

ORCID iD

Wiebke Sondermann http://orcid.org/0000-0002-3684-3523

\section{REFERENCES}

1 Schedlowski M, Enck P, Rief W, et al. Neuro-Bio-Behavioral mechanisms of placebo and nocebo responses: implications for clinical trials and clinical practice. Pharmacol Rev 2015;67:697-730.

2 Benedetti F. Placebo effects: from the neurobiological paradigm to translational implications. Neuron 2014;84:623-37.

3 Ashar YK, Chang LJ, Wager TD. Brain mechanisms of the placebo effect: an affective appraisal account. Annu Rev Clin Psychol 2017;13:73-98

4 Colloca L, Benedetti F. Placebos and painkillers: is mind as real as matter? Nat Rev Neurosci 2005;6:545-52.

5 Bingel U, Wanigasekera V, Wiech $\mathrm{K}$, et al. The effect of treatment expectation on drug efficacy: imaging the analgesic benefit of the opioid remifentanil. Sci Transl Med 2011;3:70ra14.

6 Rutherford BR, Wall MM, Brown PJ, et al. Patient expectancy as a mediator of placebo effects in antidepressant clinical trials. Am J Psychiatry 2017;174:135-42.

7 Augustin M, Reich K, Glaeske G, et al. Co-Morbidity and agerelated prevalence of psoriasis: analysis of health insurance data in Germany. Acta Derm Venereol 2010;90:147-51.

8 Parisi R, Symmons DPM, Griffiths CEM, et al. Global epidemiology of psoriasis: a systematic review of incidence and prevalence. $J$ Invest Dermatol 2013;133:377-85.

9 Hawro M, Maurer M, Weller K, et al. Lesions on the back of hands and female gender predispose to stigmatization in patients with psoriasis. J Am Acad Dermatol 2017;76:648-54.

10 Koo J, Marangell LB, Nakamura M, et al. Depression and suicidality in psoriasis: review of the literature including the cytokine theory of depression. J Eur Acad Dermatol Venereol 2017;31:1999-2009.

11 Patel N, Nadkarni A, Cardwell LA, et al. Psoriasis, depression, and inflammatory overlap: a review. Am J Clin Dermatol 2017;18:613-20.

12 Boehncke W-H, Boehncke S, Tobin A-M, et al. The 'psoriatic march': a concept of how severe psoriasis may drive cardiovascular comorbidity. Exp Dermatol 2011;20:303-7.

13 Augustin M, Krüger K, Radtke MA, et al. Disease severity, quality of life and health care in plaque-type psoriasis: a multicenter crosssectional study in Germany. Dermatology 2008;216:366-72.

14 Finlay AY, Kelly SE. Psoriasis--an index of disability. Clin Exp Dermatol 1987;12:8-11.

15 Malhotra SK, Mehta V. Role of stressful life events in induction or exacerbation of psoriasis and chronic urticaria. Indian J Dermato Venereol Leprol 2008;74:594-9.

16 Kiecolt-Glaser JK, Heffner KL, Glaser R, et al. How stress and anxiety can alter immediate and late phase skin test responses in allergic rhinitis. Psychoneuroendocrinology 2009;34:670-80.

17 Enck P, Bingel U, Schedlowski M. The placebo response in medicine: minimize. maximize or personalize? Nat Rev Drug Discov 2013;12:191-204.

18 van Laarhoven AIM, van der Sman-Mauriks IM, Donders ART, et al. Placebo effects on itch: a meta-analysis of clinical trials of patients with dermatological conditions. J Invest Dermatol 2015;135:1234-43.

19 Lynde CW, Poulin Y, Vender R, et al. Interleukin 17A: toward a new understanding of psoriasis pathogenesis. J Am Acad Dermatol 2014;71:141-50.

20 Rendon A, Schäkel K. Psoriasis pathogenesis and treatment. Int J Mol Sci 2019;20. doi:10.3390/ijms20061475. [Epub ahead of print: 23 Mar 2019].

21 Nestle FO, Kaplan DH, Barker J. Psoriasis. N Engl J Med 2009;361:496-509.

22 Girolomoni G, Strohal R, Puig L, et al. The role of IL-23 and the IL-23/ $\mathrm{T}_{\mathrm{H}} 17$ immune axis in the pathogenesis and treatment of psoriasis. $J$ Eur Acad Dermatol Venereol 2017;31:1616-26.

23 Langley RG, Elewski BE, Lebwohl M, et al. Secukinumab in plaque psoriasis--results of two phase 3 trials. $N$ Engl J Med 2014;371:326-38.

24 Marzo-Ortega H, Sieper J, Kivitz A, et al. Secukinumab and sustained improvement in signs and symptoms of patients with active ankylosing spondylitis through two years: results from a phase III study. Arthritis Care Res 2017;69:1020-9.

25 Mease PJ, Mclnnes IB, Kirkham B, et al. Secukinumab inhibition of interleukin-17A in patients with psoriatic arthritis. $N$ Engl J Med 2015;373:1329-39.

26 Deodhar A, Mease PJ, Mclnnes IB, et al. Long-Term safety of secukinumab in patients with moderate-to-severe plaque psoriasis, psoriatic arthritis, and ankylosing spondylitis: integrated pooled clinical trial and post-marketing surveillance data. Arthritis Res Ther 2019;21:111.

27 Blauvelt A, Shi N, Zhu B, et al. Comparison of health care costs among patients with psoriasis initiating ixekizumab, Secukinumab, or adalimumab. J Manag Care Spec Pharm 2019;25:1366-76.

28 Hadamitzky M, Engler H, Schedlowski M. Learned immunosuppression: extinction, renewal, and the challenge of reconsolidation. J Neuroimmune Pharmacol 2013;8:180-8.

29 Ader R, Mercurio MG, Walton J, et al. Conditioned pharmacotherapeutic effects: a preliminary study. Psychosom Med 2010;72:192-7.

30 Finniss DG, Kaptchuk TJ, Miller F, et al. Biological, clinical, and ethical advances of placebo effects. Lancet 2010;375:686-95.

31 Forsberg JT, Martinussen M, Flaten MA. The placebo analgesic effect in healthy individuals and patients: a meta-analysis. Psychosom Med 2017;79:388-94.

32 Peciña M, Zubieta J-K. Molecular mechanisms of placebo responses in humans. Mol Psychiatry 2015;20:416-23.

33 Bartels DJP, van Laarhoven AIM, Haverkamp EA, et al. Role of conditioning and verbal suggestion in placebo and nocebo effects on itch. PLoS One 2014;9:e91727.

34 van Laarhoven AIM, Vogelaar ML, Wilder-Smith $\mathrm{OH}$, et al. Induction of nocebo and placebo effects on itch and pain by verbal suggestions. Pain 2011;152:1486-94.

35 Papoiu ADP, Wang H, Coghill RC, et al. Contagious itch in humans: a study of visual 'transmission' of itch in atopic dermatitis and healthy subjects. Br J Dermatol 2011;164:1299-303.

36 McRae C, Cherin E, Yamazaki TG, et al. Effects of perceived treatment on quality of life and medical outcomes in a double-blind placebo surgery trial. Arch Gen Psychiatry 2004;61:412-20.

37 Schaefer M, Sahin T, Berstecher B. Why do open-label placebos work? A randomized controlled trial of an open-label placebo induction with and without extended information about the placebo effect in allergic rhinitis. PLoS One 2018;13:e0192758.

38 Jockenhöfer F, Knust C, Benson S, et al. Influence of placebo effects on quality of life and wound healing in patients with chronic venous leg ulcers. J Dtsch Dermatol Ges 2020;18:103-9.

39 Ambikaibalan D, Quaade AS, Halling A-S, et al. Placebo response in phase 3 trials of systemic therapies for moderate-to-severe plaque psoriasis: a systematic review and meta-analysis. Dermatology 2021;237:1-8.

40 Montgomery AA, Peters TJ, Little P. Design, analysis and presentation of factorial randomised controlled trials. BMC Med Res Methodol 2003;3:26.

41 Papp KA, Langley RG, Sigurgeirsson B, et al. Efficacy and safety of secukinumab in the treatment of moderate-to-severe plaque psoriasis: a randomized, double-blind, placebo-controlled phase II dose-ranging study. Br J Dermatol 2013;168:412-21.

42 Hadamitzky M, Lückemann L, Pacheco-López G, et al. Pavlovian conditioning of immunological and neuroendocrine functions. Physiol Rev 2020;100:357-405.

43 Kirchhof J, Petrakova L, Brinkhoff A, et al. Learned immunosuppressive placebo responses in renal transplant patients. Proc Natl Acad Sci U S A 2018;115:4223-7.

44 Paul C, Lacour J-P, Tedremets L, et al. Efficacy, safety and usability of secukinumab administration by autoinjector/pen in psoriasis: a randomized, controlled trial (JUNCTURE). J Eur Acad Dermatol Venereol 2015;29:1082-90.

45 Nast A, Altenburg A, Augustin M, et al. German S3-Guideline on the treatment of Psoriasis vulgaris, adapted from EuroGuiDerm - Part 1: Treatment goals and treatment recommendations. J Dtsch Dermatol Ges 2021;19:934-150.

46 Rief W, Glombiewski JA, Barsky AJ. Generic assessment of side effects, 2009. Available: www.GASE-scale.com

47 Finlay AY, Khan GK. Dermatology Life Quality Index (DLQI)-a simple practical measure for routine clinical use. Clin Exp Dermatol 1994;19:210-6.

48 Cohen S, Kamarck T, Mermelstein R. A global measure of perceived stress. J Health Soc Behav 1983;24:385-96.

49 Nater UM, Rohleder N. Salivary alpha-amylase as a non-invasive biomarker for the sympathetic nervous system: current state of research. Psychoneuroendocrinology 2009;34:486-96. 
50 Pruessner JC, Wolf OT, Hellhammer DH, et al. Free cortisol levels after awakening: a reliable biological marker for the assessment of adrenocortical activity. Life Sci 1997;61:2539-49.

51 Stalder T, Kirschbaum C, Kudielka BM, et al. Assessment of the cortisol awakening response: expert consensus guidelines. Psychoneuroendocrinology 2016;63:414-32.

52 Laux L, Hock M, Bergner-Köther R. Das State-trait-AngstDepressions-Inventar: STADI; manual. Göttingen: Hogrefe, 2013.

53 Strobel A, Beauducel A, Debener S, et al. Eine deutschsprachige version des BIS/BAS-Fragebogens von carver und white. Zeitschrift für Differentielle und Diagnostische Psychologie 2001:22:216-27.

54 Barsky AJ, Wyshak G, Klerman GL. The somatosensory amplification scale and its relationship to hypochondriasis. J Psychiatr Res 1990;24:323-34.
55 Rammstedt B, John OP. Measuring personality in one minute or less: a 10-item short version of the big five inventory in English and German. J Res Pers 2007;41:203-12.

56 Shedden-Mora M, Alberts J, Petrie K, et al. The treatment expectation questionnaire (TEX-Q) - A generic multidimensional scale measuring patients' treatment expectations. J Psychosom Res 2019;121:129-30.

57 Faul F, Erdfelder E, Lang A-G, et al. G*Power 3: a flexible statistical power analysis program for the social, behavioral, and biomedical sciences. Behav Res Methods 2007;39:175-91.

58 Huque MH, Carlin JB, Simpson JA, et al. A comparison of multiple imputation methods for missing data in longitudinal studies. BMC Med Res Methodol 2018;18:168.

59 Chan A-W, Tetzlaff JM, Altman DG, et al. Spirit 2013 statement: defining standard protocol items for clinical trials. Ann Intern Med 2013;158:200-7. 